\title{
DISEÑO DE UNA MAQUETA DE BAJO COSTE PARA SHM
}

\author{
Diego García Pérez, Ignacio Díaz Blanco, Ana González Muñiz, Abel A. Cuadrado Vega \\ Área de Ingeniería de Sistemas y Automática, Universidad de Oviedo \\ Campus universitario de Gijón s/n, 33204 \\ idiaz@uniovi.es, diegogarcia@isa.uniovi.es, anaglezmuniz@gmail.com, cuadrado@isa.uniovi.es
}

\section{Resumen}

En este artículo se presenta el diseño preliminar de una maqueta para la experimentación en sistemas de monitorización de salud estructural (structural health monitoring, SHM). La estructura propuesta es fácilmente adaptable al experimento a realizar y está construida a partir de materiales de bajo coste y piezas de plástico impresas en $3 D$. Para monitorizar el estado de la estructura, se sugiere un sistema de adquisición de datos compuesto por un Arduino y varios acelerómetros de bajo coste dispuestos a lo largo de la estructura. Varias visualizaciones de los datos extraídos demuestran que, con este simple diseño, se puede conseguir información relevante y repetible sobre la dinámica de la estructura.

Palabras clave: monitorización de salud estructural, métodos basados en vibración, impresión 3D, Arduino.

\section{INTRODUCCIÓN}

Los sistemas de monitorización de salud estructural (structural health monitoring, SHM) son aquellos capaces de detectar fallos o defectos en sistemas mecánicos (máquinas, aeronaves, grandes edificaciones, puentes, etc.) mediante la monitorización de las desviaciones del sistema con respecto a un estado óptimo de funcionamiento, generalmente el inicial [1, 2]. Existen diferentes métodos para caracterizar el estado del sistema [3, 4, pero uno de los enfoques más consolidados en la literatura, y en el que este trabajo se apoya, es la medición de las aceleraciones del sistema tras una excitación de entrada. A este enfoque se le conoce como vibration-based methods [5] y su objetivo es elaborar modelos del sistema a partir de técnicas de análisis modal.

Según [6, el desarrollo de métodos basados en análisis de vibraciones puede descomponerse en cuatro tareas:

1. Evaluación operativa.

2. Adquisición de datos y su normalización.
3. Extracción de características.

4. Desarrollo de modelos.

De entre estas fases, la adquisición de datos supone un gran obstáculo, debido al enorme coste asociado a sensorizar un sistema mecánico real y a que, por motivos de seguridad, muchos de los escenarios de fallo no pueden ser simulados en sistemas en funcionamiento. Por estas razones, muchos trabajos en SHM [7, 8, 9] desarrollan maquetas a escala, en las que simular fallos de forma segura y con un coste reducido.

A pesar de los beneficios asociados al uso de una maqueta como fuente de los datos, en ocasiones estas contienen elementos hechos a medida, costosos o que requieren de un fuerte conocimiento interdisciplinar, lo que las hacen poco reproducibles. La motivación del presente trabajo es el desarrollo de un nuevo diseño basado en tecnologías de código abierto y de reducido coste, que facilite la construcción de este tipo de banco de ensayos por otros grupos.

Los principales aportes de nuestro trabajo para la consecución de este objetivo son: 1) la construcción de una estructura con la ayuda de las nuevas tecnologías de fabricación aditiva, comúnmente conocidas como impresión $3 \mathrm{D}, 2$ ) sustitución de los costosos acelerómetros de alta frecuencia utilizados en trabajos previos 9, por acelerómetros basados en tecnología $M E M S$ mucho más asequibles y 3) el uso del reconocible Arduino MEGA 2560 [10] como una tarjeta de adquisición de datos de código abierto.

El resto del artículo está organizado de la siguiente manera. En el apartado de 2 se expone detalladamente los diferentes elementos que conforman la maqueta; en el apartado 3 se describe la configuración elegida para una captura preliminar de datos; en el apartado 4 se presenta una pequeña exploración de la repetibilidad y la calidad de la información recopilada; por último, en el apartado 5 se discuten los resultados obtenidos y posibles mejoras de nuestro diseño. 


\section{Diseño}

La maqueta propuesta está formada por los siguientes elementos: 1) la estructura, 2) el sistema de excitación, 3) el sistema de adquisición de datos y 4) la automatización de los ensayos. Para cada uno de estos elementos se proponen soluciones fácilmente reproducibles y que permiten rápidas reconfiguraciones de los mismos.

\subsection{Estructura}

En el diseño de la estructura se ha huido de grandes estructuras metálicas hechas a medida y se ha optado por estructuras desmontables formadas por dos elementos fundamentales:

- Nudos. Son los elementos que mantienen unida la estructura y definen su forma final. En diseños anteriores [9, los nudos son piezas metálicas como pernos o abrazaderas, los cuales limitan enormemente las posibles configuraciones. Nuestra propuesta consiste en remplazar los nudos metálicos por piezas de plástico impresas en 3D. Gracias a la impresión 3D, podemos diseñar y construir nudos con geometrías complejas que nos permiten ensamblar multitud de estructuras, tales como mallas, celosías o armazones.

- Barras. Es el elemento que une varios nudos entre sí. Típicamente suelen ser de materiales rígidos capaces de soportar las tensiones que sufre la estructura. Siguiendo nuestro objetivo de hacer la maqueta lo más asequible posible, las barras serán varillas roscadas de métrica $M 6$ cuyo coste aproximado es de $1 € \backslash m$. A la ventaja económica se suma que son fáciles de cortar a medida.

Para poder construir una estructura con estos dos elementos es necesario definir un mecanismo de fijación entre ellos que, además de garantizar una conexión sólida, permita deshacer la unión sin mucho esfuerzo. Experimentalmente se decidió que la unión más robusta y versátil es dotar al nudo de un roscado interno donde se pueda atornillar la varilla roscada como se ilustra en la Figura 1.

De entre todas las posibles estructuras que nos permite montar el mecanismo de barra/nudo, decidimos centrar nuestras primeras pruebas en una estructura tipo celosía como la viga Warren mostrada en la Figura 2, En [11, se encuentran disponibles todos los modelos $3 \mathrm{D}$ de los nudos necesarios para montar una estructura como la de la figura. Las barras utilizadas para su montaje fueron de longitud $300 \mathrm{~mm}$. Aunque la estructura propuesta esté lejos de simular una estructura real,

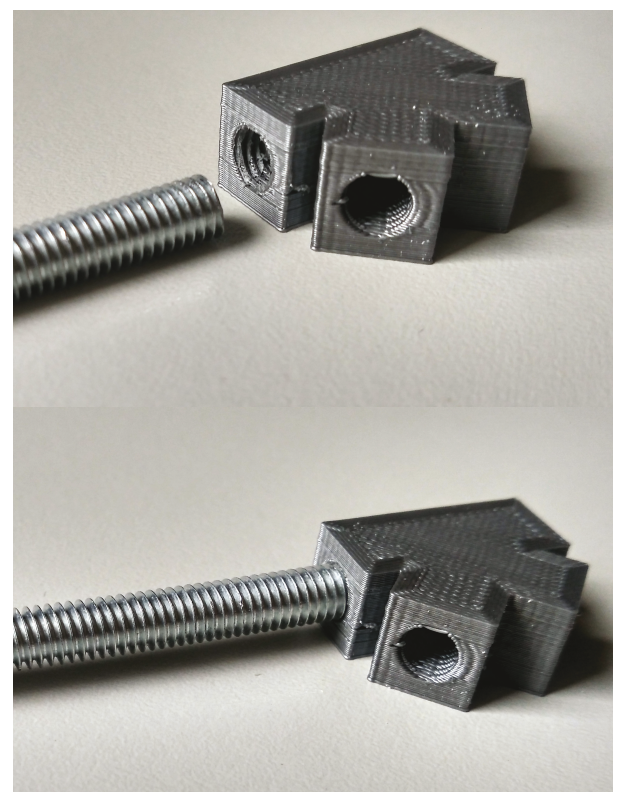

Figura 1: Unión roscada entre barra y nudo.

es una configuración sencilla con la que probar el resto de elementos de nuestra maqueta.

\subsection{Sistema de excitación}

Un modelo SHM basado en vibraciones requiere poder medir cómo responde el sistema ante excitaciones de entrada, que pueden ser propias a él, como por ejemplo un vehículo circulando por un puente, o forzadas acoplando a la estructura un sistema de excitación [12. En nuestro caso, el sistema carece de excitaciones intrínsecas por lo que deben inducirse mediante alguno de los múltiples métodos del estado del arte [9, 13, 14. Muchos de estos métodos introducen vibraciones de muy alta frecuencia 14, las cuales obligarían a instalar acelerómetros con un gran ancho de banda que alejaría nuestra maqueta del objetivo principal, la reproducibilidad. Por ello, creemos que estos dos métodos de excitación de baja frecuencia son los más apropiados:

- Percutor electromecánico. El percutor, como se muestra en la Figura 3, lo constituye un solenoide DC de $12 \mathrm{~V}$ 15. Cuando la bobina del solenoide está energizada atrae el eje hacia el núcleo y cuando la corriente dentro de la bobina es cero, el muelle arrastra el eje hasta su posición de reposo. De esta manera se puede conseguir un movimiento lineal que introduce una excitación de tipo impulso, perfecta para obtener la respuesta dinámica característica de nuestro sistema.

- Vibrador de masa excéntrica. Este tipo de actuadores consisten en un motor con una masa 


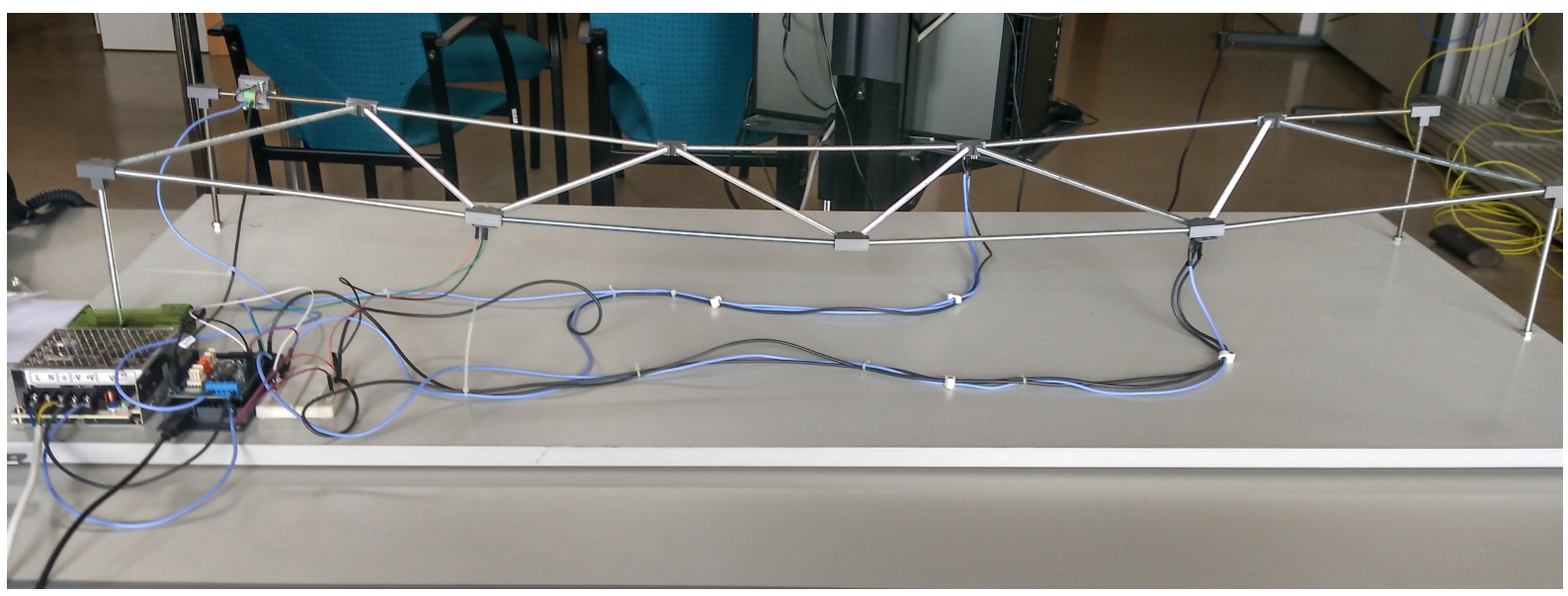

Figura 2: Estructura tipo viga Warren.

excéntrica acoplada a su eje que, al rotar, induce una vibración. En nuestro caso, se ha elegido un motor DC 16] de $9 \mathrm{~V}$ como el mostrado en la Figura 4, el cual se extrajo del mando de una videoconsola. Los vibradores de masa excéntrica introducen una entrada tipo senoidal cuya frecuencia y magnitud dependen de la velocidad de giro del motor. Esto permite introducir barridos frecuenciales [17] a nuestra estructura y realizar un análisis dinámico en frecuencia de nuestro sistema.

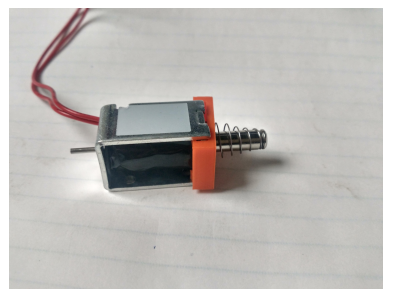

Figura 3: Percutor electromecánico.

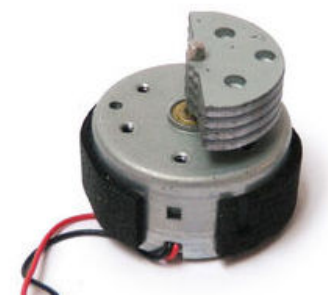

Figura 4: Vibrador de masa excéntrica. Está formado por un motor DC con una masa excéntrica acoplada a su eje.

Para acoplar estos dos actuadores se modelaron en 3D dos piezas de sujeción que, al igual que los nudos, los archivos correspondientes a sus modelos 3D pueden encontrarse en [11. Estas dos piezas de agarre fueron diseñadas para ser acopladas firmemente en cualquier barra de la estructura y en cualquier orientación. Esto permite al usuario configurar, sin mucho esfuerzo, la dirección de la vibración introducida a la estructura. En la Figura 5 se muestran los dos actuadores montados en la estructura.

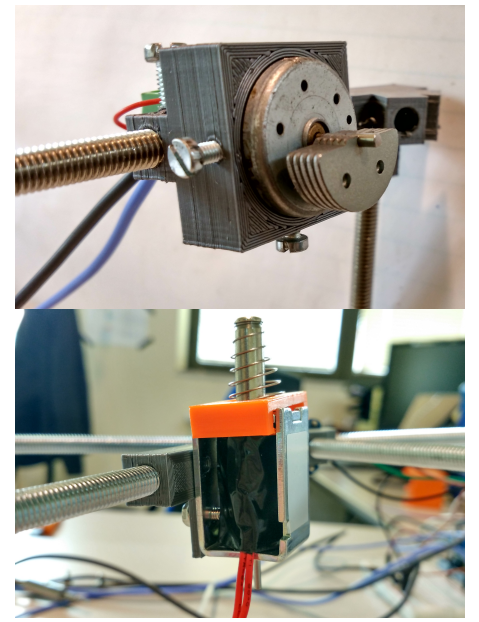

Figura 5: Actuadores fijados a la estructura mediante sus piezas de sujeción.

Los dos actuadores propuestos son elementos que, a pesar de ser de baja potencia, un microcontrolador de bajo coste como el Arduino no puede manejar sin ayuda de un pre-accionador. El Arduino motor shield [18] será el driver encargado de gestionar la entrega de potencia, desde la fuente de alimentación de $12 \mathrm{~V}$ a nuestros actuadores.

\subsection{Adquisición de datos}

Una vez definida la estructura y el sistema de excitación, el siguiente paso es capturar adecuadamente la respuesta del sistema. El sistema de medición propuesto puede dividirse en: 1) acelerómetros, 2) tarjeta de adquisición de datos y 3 ) envío de la 


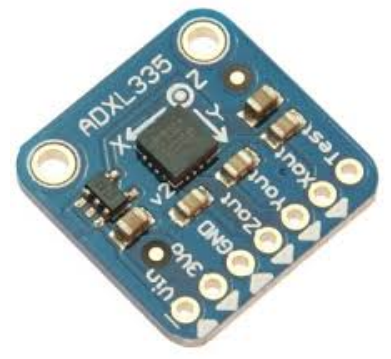

Figura 6: Acelerómetros MEMS ADXL335.

información.

Acelerómetros. Para recoger la respuesta modal a lo largo de una gran estructura, los sistemas de monitorización de salud estructural no utilizan la información de un único acelerómetro, sino que parten de una mapa de aceleraciones capturado por un array de sensores [17, 12, 9, 4, correctamente situados a lo largo de la estructura. Decidir el número de acelerómetros y su posición es, por sí solo, un campo de estudio en SHM [7] y en ocasiones requiere varias iteraciones. Es por estas razones que los acelerómetros elegidos han de ser manejables, asequibles y fáciles de instalar. Los acelerómetros MEMS ADXL335 19] de 3 grados de libertad, como el mostrado en la Figura 6, reúnen todos estos requisitos, puesto que son acelerómetros de bajo coste y de tamaño reducido.

Tarjeta de adquisición de datos. Como ya se adelantó en las secciones 1 y 2.2 , el microcontrolador que gobierna nuestro banco de pruebas es el $A r$ duino Mega 2560 [10]. La tarea del Arduino dentro de nuestro sistema de adquisición de datos es la de ser una tarjeta de adquisición de datos flexible y barata. A través de sus convertidores ADC de 10 bits, el Arduino leerá las señales de salida de los ADXL 335 a una frecuencia de muestreo constante. Para garantizar esta frecuencia de muestreo, la lectura analógica se implementa en una interrupción temporal, que se ejecuta de forma cíclica tras el desbordamiento de un temporizador. La máxima frecuencia de muestreo del Arduino viene marcada por el tiempo de ejecución de la rutina de interrupción, el cual depende del tiempo de conversión y del número de aceleraciones a medir. Así, la máxima frecuencia de muestreo conseguida para 6 aceleraciones ha sido de $10 \mathrm{kHz}$.

Envío de la información. De nada sirve leer las aceleraciones, si estas no se almacenan para su posterior análisis. La memoria RAM del Arduino no tiene capacidad suficiente para almacenar toda la información generada en un ensayd ${ }^{1}$. Por este

\footnotetext{
${ }^{1}$ Suponiendo la lectura de 6 medidas tipo int ( 2 bytes) a $1 k H z$, la memoria SRAM de $8 K B$ desbordaría antes del primer segundo de ensayo.
}

motivo, el Arduino debe enviar mediante su puerto serie las mediciones de los sensores a un PC con memoria suficiente para almacenar nuestros ensayos. El ancho de banda del puerto serie del Arduino MEGA supone una nueva limitación en la frecuencia de muestreo, lo que nos obliga a reducir nuestra tasa de muestreo de $10 \mathrm{kHz}$ a $1 \mathrm{kHz}$.

Una vez que se ha realizado el ensayo y se han enviado al PC todos los datos del mismo, estos pueden ser guardados en archivos tipo .csv, .json o en otro tipo de sistemas de almacenamiento estructurado de datos como bases de datos.

\subsection{Automatización de los ensayos}

La tendencia actual en el análisis de datos, motivada en parte por el impulso de las TIC y el abaratamiento de los sensores, es hacer uso de grandes cantidades de datos para mejorar el rendimiento de los algoritmos. Por eso no es de extrañar que en trabajos como el presente se propongan métodos para automatizar la captura de la información, que agilicen el proceso de obtención de grandes conjuntos de datos. Así, en nuestro caso concreto, una vez configurada la comunicación serie entre Arduino y PC, no resulta demasiado complicado automatizar los ensayos desde un entorno de programación de alto nivel como Matlab [20] o Python [21. Estos entornos permiten, sin tener un gran nivel de programación, realizar interfaces con nuestro Arduino en los que el usuario puede definir parámetros del ensayo (duración, frecuencia de muestreo, etc.), elegir el tipo de excitación o el número de ensayos a realizar.

\section{Definición de los ensayos}

En el apartado anterior se remarcó que nuestra propuesta de maqueta, más que una estructura con una forma fija, es un diseño flexible que puede adaptarse para simular diferentes estructuras. Además, algunas de las tecnologías utilizadas, como la impresión 3D, permiten simular multitud de fallos estructurales. En la siguiente lista se presentan todos los elementos que el usuario puede variar para obtener la configuración física que necesite:

- El tipo de estructura. Gracias al sistema nudo/barra podemos idear multitud de modelos estructurales, tales como: vigas en celosía, estructuras en armazón, mallados, etc.

- Dimensiones. Variando el número de nudos o la longitud de las barras es posible adecuar las dimensiones de la estructura a nuestros intereses.

- La disposición y el número de los aceleróme- 


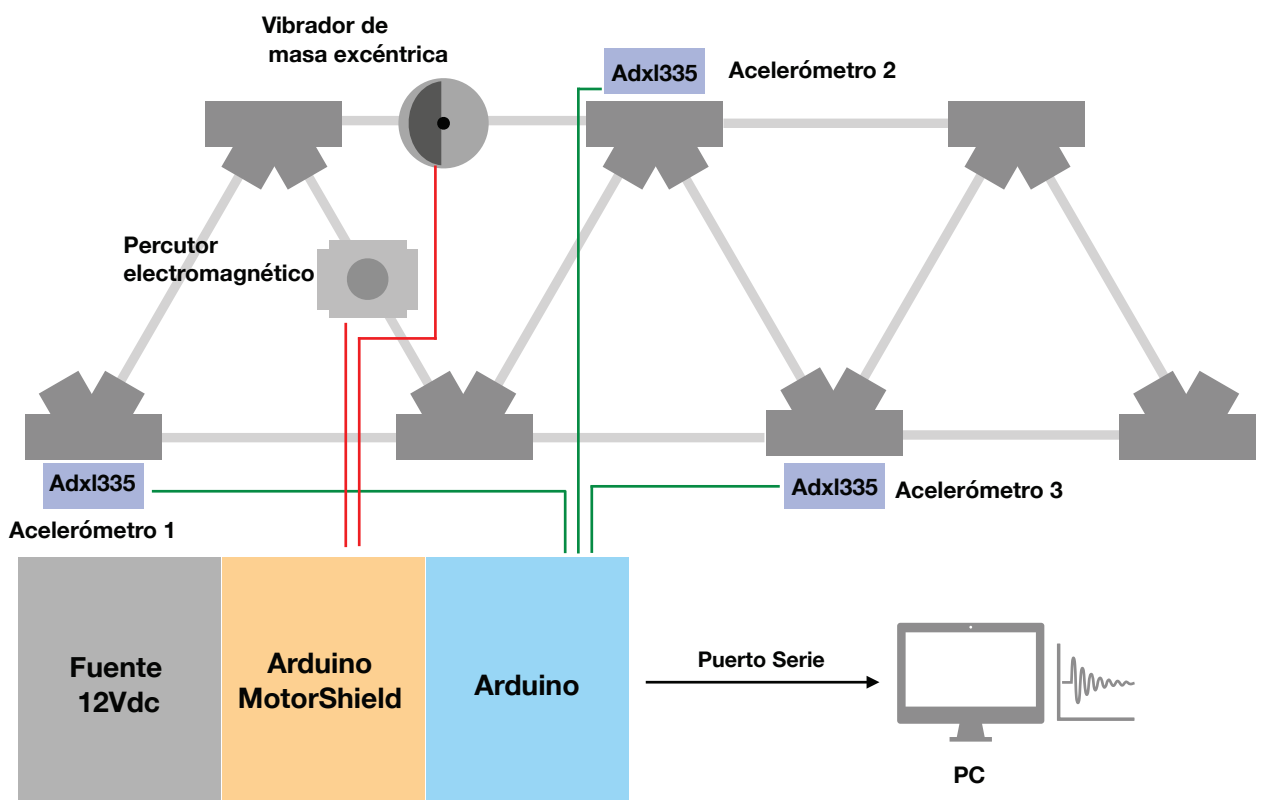

Figura 7: Esquema explicativo de la primera versión funcional de la maqueta.

tros. Como se explicó en 2.3 , poder redefinir las posiciones de los acelerómetros es un aspecto relevante en la monitorización de salud estructural.

Plantear ensayos que simulen situaciones anómalas, en muchas ocasiones, implica el deterioro deliberado de elementos estructurales con la consiguiente pérdida de dinero. Nuestro enfoque de bajo coste y la tecnología de impresión 3D supone una gran ventaja en este aspecto. La siguiente lista es una representación de fallos que podemos llegar a inducir:

1. Barra floja o suelta. Desatornillar una de las barras, llegando, incluso, a soltarse.

2. Nudo defectuoso. La impresión 3D nos permite fabricar nudos con defectos internos, tales como: grietas, cambios en la densidad del material o errores dimensionales.

3. Reducción de la sección de las barras. La sección de una barra puede ser reducida con un pequeño corte localizado.

De entre todas las combinaciones posibles de estructuras y fallos, hemos elegido una configuración de partida como la mostrada en el esquema de la Figura 7. A la estructura tipo celosía se han acoplado 3 acelerómetros, los cuales recogen las aceleraciones verticales que experimenta el sistema durante el ensayo. Para generar la excitación de entrada se instala un vibrador de masa excéntrica. La respuesta del sistema al barrido en frecuencia inducido por el motor es medida a una frecuencia de muestreo fija de $1 \mathrm{kHz}$. En cuanto a los estados de fallo, solo se contemplan los tipos de fallo relacionados con las barras, es decir, los fallos $1 \mathrm{y}$ 3 .

\section{Exploración preliminar de los datos}

Aunque el objetivo de este trabajo no es el desarrollo de modelos SHM, sino el diseño de una maqueta de ensayos, creemos que es interesante presentar una sencilla exploración visual de los primeros datos capturados.

Partiendo de una configuración como la mostrada en el esquema de la Figura 7, se realizan 3 ensayos iguales para cada uno de los siguientes estados o clases de la maqueta:

1. Estado normal.

2. Con una barra suelta.

3. Con una barra parcialmente seccionada.

Los datos obtenidos para los nueve experimentos se presentan en la Figura 8, En la figura se puede observar cómo a lo largo del barrido en frecuencia aparecen diferentes resonancias en distintos puntos de la estructura. Esta información es una firma del estado de la estructura, ya que estas resonancias guardan relación con los polos del sistema. Si el sistema sufre algún cambio, como por ejemplo el desprendimiento de una barra, la localización 

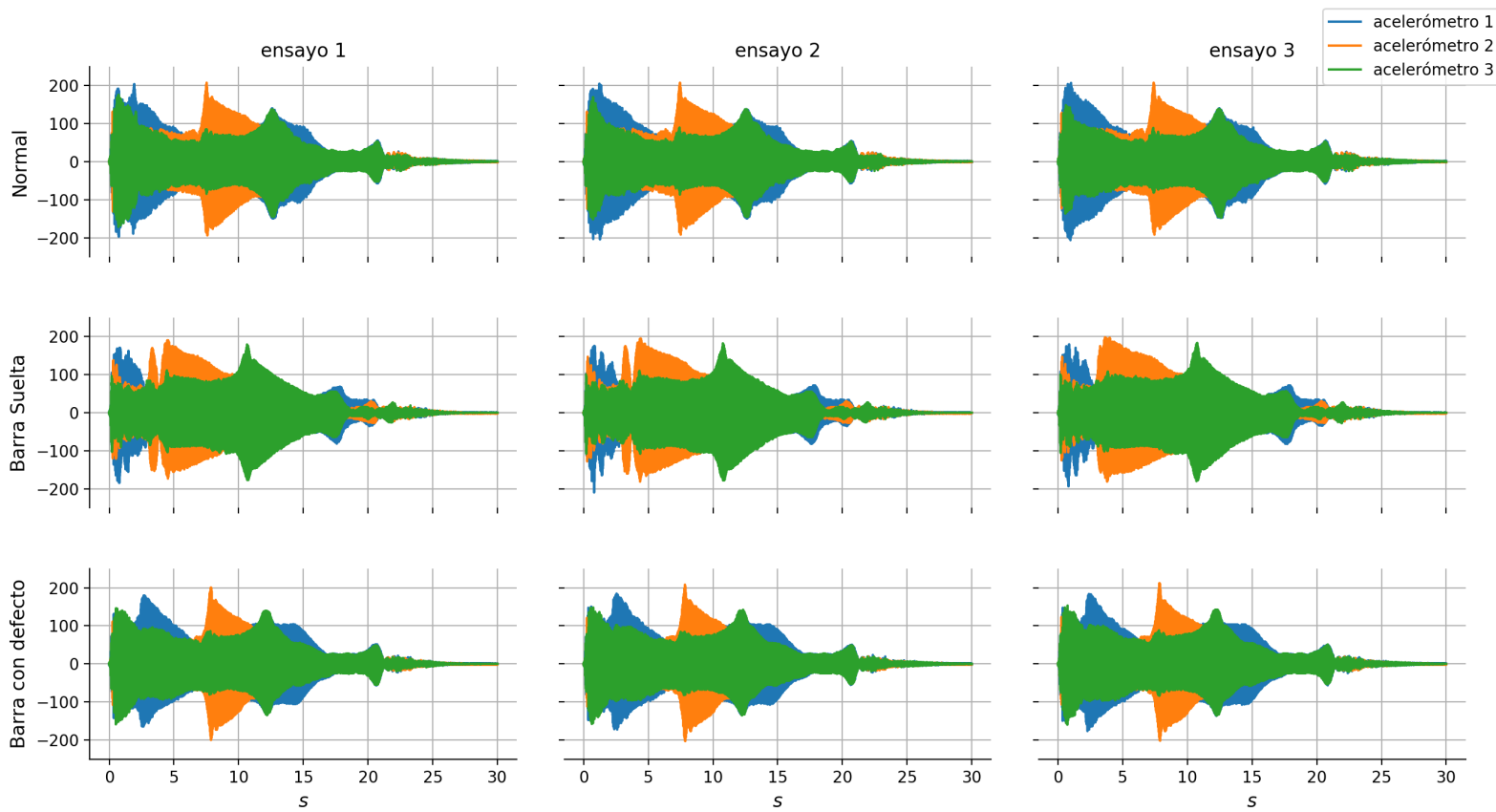

Figura 8: Aceleraciones capturadas en los nueve ensayos realizados. Las gráficas de las aceleraciones se presentan ordenadas por clase y ensayo. Cada color representa uno de los tres acelerómetros instalados en la estructura.
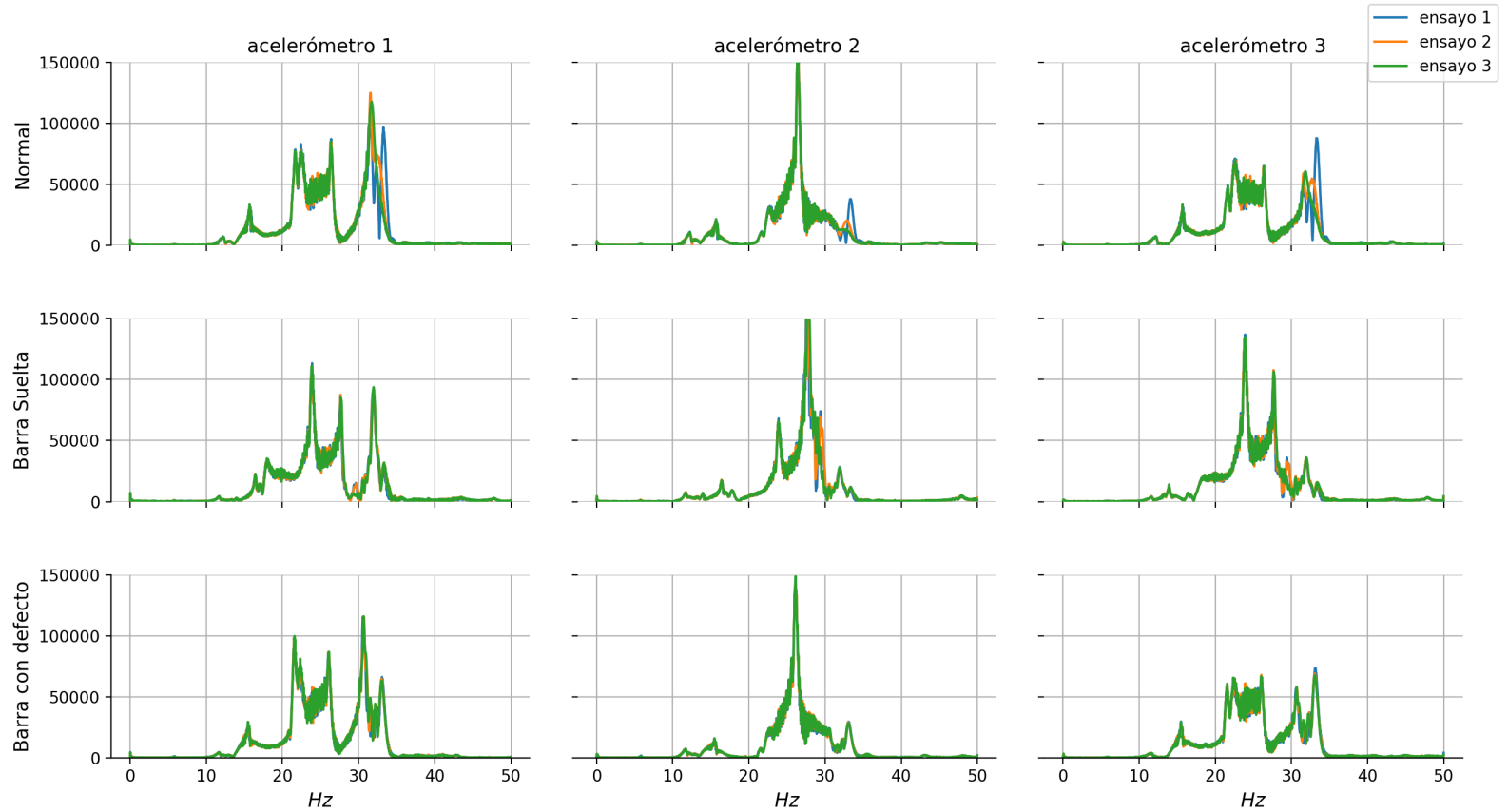

Figura 9: FFT de los 9 experimentos ordenados por clase y acelerómetro.

de estos polos varía, resultando en una respuesta dinámica completamente diferente a la observada en el estado normal. Estas diferencias pueden observarse en los ensayos realizados con una barra suelta, en los que el mapa de aceleraciones revela un patrón resonancias muy distinto al registrado en los ensayos de estado normal.

Otro de los aspectos críticos en un banco de ensa- yos como este es garantizar que los experimentos en las mismas condiciones sean lo más repetibles posibles. En la propia Figura 8, ya se puede observar esta repetibilidad, pero creemos que una vista de los ensayos en el dominio de la frecuencia es más adecuada. Así, en la Figura 9 se presentan 
las transformadas de Fourie1 2 de los nueve ensayos ordenados por clase y acelerómetro para la banda de frecuencias de 0 a $40 \mathrm{~Hz}$. Dentro de todo el espectro en frecuencia, la banda presentada es la que más información porta y, en ella, se puede observar que los módulos de las componentes de cada ensayo están prácticamente superpuestos, lo que demuestra que los ensayos son bastante repetibles. En este experimento, también se aprecia cómo los experimentos con una barra suelta son claramente diferentes en frecuencia al estado normal, mientras que las diferencias entre la barra con defecto y el estado normal son menos evidentes. Una barra con un defecto tan pequeño como un corte localizado probablemente transmita mejor las vibraciones de baja frecuencia y perturbe las altas frecuencias. En la Figura 10 se observan estas pequeñas perturbaciones en el acelerómetro 2.

Este primer análisis exploratorio confirma que las aceleraciones recogidas reflejan fielmente el estado del sistema y, por tanto, contienen información útil a la hora de caracterizar el estado del sistema.

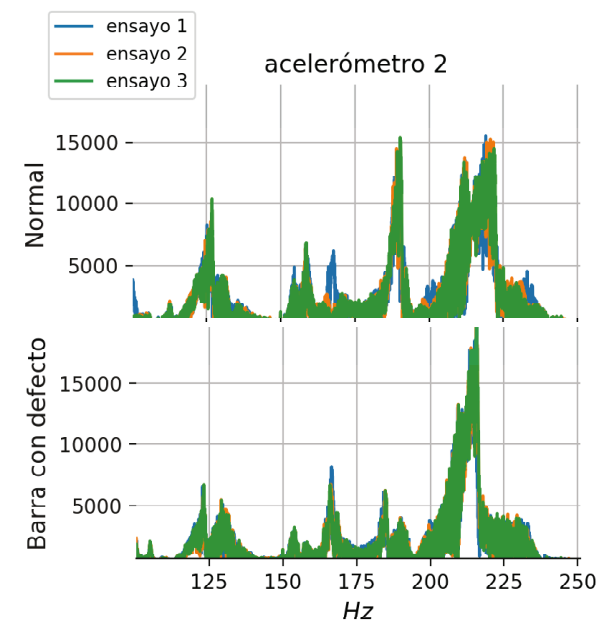

Figura 10: Módulo de la transformada de Fourier del acelerómetro 2, para los estados normales y con una barra defectuosa.

\section{Conclusiones}

Este trabajo presenta un diseño flexible y fácil de replicar de una maqueta para experimentos en el campo de la monitorización de la salud estructural. El sistema nudo/barra propuesto permite a nuestra maqueta simular multitud de escenarios reales, sin más esfuerzo que atornillar y desatorni-

\footnotetext{
${ }^{2}$ El paso al dominio de la frecuencia se llevo a cabo mediante una implementación en Python del método Fast Fourier Transform (FFT).
}

llar barras. A esta flexibilidad se añade que todos los materiales utilizados son de bajo coste, incluido el controlador y los sensores. Gracias a ello, la primera versión funcional fue construida con un presupuesto menor de $100 €$.

A pesar de la flexibilidad y de la reproducibilidad, nuestro diseño también presenta algunas limitaciones. La más importante es que el número de acelerómetros es un factor limitante. Al aumentar el número de aceleraciones medidas se reduce considerablemente la frecuencia de muestreo, debido a que el ancho de banda del puerto serie de nuestro Arduino MEGA no es suficiente para enviar todos los datos. Una posible solución para este problema es sustituir el Arduino MEGA por otra placa más potente como el Arduino DUE, cuyo puerto serie tiene un mayor ancho de banda. Otra de las desventajas de nuestro diseño es que para fabricar los nudos se necesita una impresora 3D, que aunque en los últimos años su uso se ha extendido, aún sigue siendo una tecnología en proceso de implantación. En el caso de no disponer de ella, una solución a tener en cuenta antes de invertir en la compra de una, es enviar los modelos 3D proporcionados a una empresa que suministre servicios de impresión en plástico.

Pese a estas limitaciones, creemos que nuestro banco de ensayos es una valiosa herramienta de experimentación para SHM capaz de recopilar información de multitud de situaciones. Esto la convierte en una fuente de datos muy asequible y recomendable para aquellos que se inicien en el desarrollo de sistemas de monitorización de salud estructural.

\section{Agradecimientos}

Este trabajo ha sido financiado por el Ministerio de Economía y Competitividad (MINECO), Programa Estatal de I+D+i Orientada a los Retos de la Sociedad y por el Fondo Europeo de Desarrollo Regional (FEDER) "Una manera de hacer Europa", bajo el proyecto de referencia DPI201569891-C2-2-R. Además, dos de los co-autores del presente trabajo disfrutan de una beca del Gobierno del Principado de Asturias a través de su programa "Severo Ochoa" de ayudas predoctorales para la investigación y docencia del Principado de Asturias. 


\section{English summary \\ DESIGN OF A LOW-COST TEST BENCH FOR SHM}

\begin{abstract}
In this article, a preliminary design of a test bench for experiments in structural health monitoring (SHM) is presented. The suggested structure can be easily adapt to the desired experiment and it is build with low-cost elements and $3 D$ printed parts. In order to monitor the structure state, we implemented a data acquisition system which consists in an Arduino board and several accelerometers installed along the structure. Finally, a brief exploration of the acquired data shows that relevant and repeatable information can be gathered from the proposed test bench.
\end{abstract}

Keywords: Structural Health Monitoring, vibration-based analysis, 3D printing technology, Arduino.

\section{Referencias}

[1] Charles R Farrar and Keith Worden. An introduction to structural health monitoring. Philosophical Transactions of the Royal Society of London A: Mathematical, Physical and Engineering Sciences, 365(1851):303315, 2007.

[2] Hoon Sohn, Charles R Farrar, Francois M Hemez, Devin D Shunk, Daniel W Stinemates, Brett R Nadler, and Jerry J Czarnecki. A review of structural health monitoring literature: 1996-2001. Los Alamos National Laboratory, USA, 2003.

[3] Jinping Ou and Hui Li. Structural health monitoring in mainland china: review and future trends. 9(3):219-231.

[4] Adam B Noel, Abderrazak Abdaoui, Tarek Elfouly, Mohamed Hossam Ahmed, Ahmed Badawy, and Mohamed S Shehata. Structural health monitoring using wireless sensor networks: A comprehensive survey. 19(3):14031423 .
[5] Scott W Doebling, Charles R Farrar, Michael B Prime, and others. A summary review of vibration-based damage identification methods. Shock and vibration digest, 30(2):91105, 1998.

[6] Charles R Farrar, Scott W Doebling, and David A Nix. Vibration-based structural damage identification. Philosophical Transactions of the Royal Society of London A: Mathematical, Physical and Engineering Sciences, 359(1778):131-149, 2001.

[7] Hao Sun and Oral Büyüköztürk. Optimal sensor placement in structural health monitoring using discrete optimization. Smart $M a$ terials and Structures, 24(12):125034, 2015.

[8] Ernesto F Castillo and Marcela A Cruchaga. Experimental vibration analysis for a $3 \mathrm{~d}$ scaled model of a three-floor steel structure. Latin American Journal of Solids and Structures, 9(5):597-613, 2012.

[9] Reza Mohammadi Ghazi, Justin G Chen, and Oral Büyüköztürk. Pairwise graphical models for structural health monitoring with dense sensor arrays. Mechanical Systems and Signal Processing, 93:578-592, 2017.

[10] Página oficial arduino mega. https://www . arduino.cc/en/Main/arduinoBoardMega Accessed: 2017-06-09.

[11] Enlace a los modelos 3d de las piezas utilizadas. https://www.thingiverse.com/ thing:3640390. Accessed: 2019-05-15.

[12] Hoon Sohn, C FARRAR, M FUGATE, and J CZARNECKI. Structural health monitoring of welded connections. Technical report, Los Alamos National Lab., NM (US), 2001.

[13] MT Bement and TR Bewley. Excitation design for damage detection using iterative adjoint-based optimization-part 1: Method development. Mechanical Systems and Signal Processing, 23(3):783-793, 2009.

[14] Victor Giurgiutiu. Structural health monitoring: with piezoelectric wafer active sensors. Elsevier, 2007.

[15] Pull action dc d-frame solenoid.

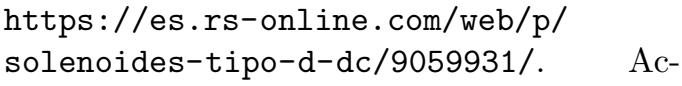
cessed: 2019-04-09.

[16] Motor de vibración para mando xbox 360. https://amzn.to/2HH80GS. Accessed: 201904-20. 
[17] Derek Nastase, Samit Ray Chaudhuri, Rebecca Chadwick, Tara C Hutchinson, Kai-Uwe Doerr, and Falko Kuester. Development and evaluation of a seismic monitoring system for building interiors - part i: Experiment design and results. IEEE Transactions on Instrumentation and Measurement, 57(2):332-344, 2008.

[18] Arduino motor shield rev3. https://store. arduino.cc/arduino-motor-shield-rev3. Accessed: 2019-05-12.

[19] Adxl335 datasheet. https://www. sparkfun.com/datasheets/Components/ SMD/adx1335.pdf | Accessed: 2019-05-12.

[20] Página oficial matlab. https://www . mathworks.com/products/matlab.html Accessed: 2019-04-23.

[21] Página oficial python. https://www. python.org/. Accessed: 2017-03-23.

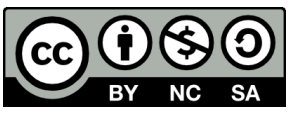

(C) 2019 by the authors. Submitted for possible open access publication under the terms and conditions of the Creative Commons Attribution CC BY-NC-SA 4.0 license (https://creativecommons.org/licenses/by-ncsa/4.0/deed.es). 